\title{
Application of the Modified Damage Index method to timber beams - I (numerical studies)
}

\author{
F.C. Choi, J. Li, B. Samali* and K. Crews \\ Center for Built Infrastructure Research, Faculty of Engineering, University of Technology Sydney \\ PO Box 123 Broadway, NSW 2007, Australia
}

\begin{abstract}
In this first part of a two-part paper, the use of two existing algorithms developed for global nondestructive evaluation to locate and evaluate localised damage in timber beams is investigated using a finite element model. These damage localisation algorithms were found, through this investigation, not to be effective in locating multiple damage scenarios and unable to evaluate the severity of damage. Hence, modifications on damage index algorithm as well as a hybrid algorithm are proposed to overcome the problems. In this study, Experimental Modal Analysis (EMA) was used as a tool to extract mode shapes for calculating the damage index in the proposed method which utilises changes in modal strain energy between the undamaged and the damaged timber beam model. The Modified Damage Index method normalises the mode shape curvature and the hybrid algorithm combines the modified index algorithm and changes in flexibility algorithms which reflect the changes of natural frequency and mode shape. Analytical evaluations were performed to compare and verify the ability of existing and modified damage localisation algorithms in locating single and multiple damage in timber beams. The Modified Damage Index algorithm and the hybrid damage algorithm are also used in the companion paper to validate the effectiveness of the methods to
\end{abstract}

\footnotetext{
* Corresponding author. Tel.: +61 2 95142023; fax: +61 295142868.

E-mail addresses: Bijan.Samali@uts.edu.au.
} 
locate and evaluate damage within timber beams by laboratory experiments.

Keywords: Damage localisation; severity estimation; timber beam; finite element method; Modified Damage Index.

\section{Introduction}

Wood is probably one of the earliest materials used by human to construct a bridge. Even though contemporary bridges take the form of concrete and steel bridges, many timber bridges are still in service. According to a document published by the Department of Transport and Regional Services [1] there are about 29,000 timber bridges in Australia. A third of these are over 50 years old and increasingly experiencing structural deficiency resulting from rot, decay, insect attack, weathering and mechanical damage. In order to avoid catastrophic failure of structures, development of a reliable condition monitoring technique for these bridge structures is of utmost importance in maintaining the integrity and safety of the structures and users.

Nondestructive evaluation (NDE) is one powerful tool which helps in assessing the structural condition of bridge structures. Many nondestructive evaluation techniques for timber structures have already been developed but most of them focus on detecting presence of defects locally by means such as visual inspection, stress wave, ultrasonic and drill resistance [2-3]. These techniques provide valuable information about the condition of a structure at given locations. However, more global information is required to assess the 'health' condition of timber structures as an entity. Global damage detection approaches are valuable means that allow the entire structural system to be assessed using changes in the global parameters without jeopardising structural 
integrity.

Over the past two decades, development of global damage detection methods, using vibration-based techniques, has attracted significant attention with respect to civil engineering structures. Kato and Shimada [4] have carried out vibration test on an existing pre-stressed concrete bridge during its demolition process to determine its vibration characteristics resulting from the deterioration. Ariyaratne et al. [5] utilised a dynamic method to test and assess the structural condition of an in-service three-span steel-concrete highway bridge. The method makes use of shift of frequency with added mass to determine its stiffness. Armstrong et al. [6] applied dynamic stiffness method, which is based on frequency response function to assess physical condition of masonry arch bridges. Salawu and Williams [7] employed full-scale vibration test on a multispan reinforced concrete bridge to monitor its dynamic characteristics before and after repair work to assess the structural condition of the bridge. Maeck and Roeck [8] used modal bending moment and modal curvature to characterise damage patterns of a pre-stressed bridge, which is gradually damaged and tested extensively. Patjawit and KanokNukulchai [9], Toksoy and Aktan [10] and Raghavendrachar and Aktan [11] have made use of the flexibility of a bridge structure to determine its condition. Among various methods, the methods that were developed based on changes in modal strain energy as an indicator of localized damage or stiffness loss in a structure have been particularly promising [12-16]. In the literature, this method is often referred to as the Damage Index (DI) method. The method was developed for application to a wide range of structural systems. Previous published studies have demonstrated the use of the DI method to localize and estimate the severity of damage within a structure using modal parameters for bridges [17-19]. Several analytical studies have been undertaken which 
verify the performance of this damage localization and severity estimation algorithm $[20]$.

However, despite an enormous number of vibration-based damage identification algorithms being proposed, and some being applied in laboratory or in the field, detailed studies on changes to dynamic behaviour of timber bridges are still in their early stages of development. Peterson and Gutkowski [21] have proposed a combined dynamic excitation system and ultrasonic inspection approach for evaluating structural integrity of timber bridges. A global nondestructive approach using impact generated frequency response functions by Morison et al. [22] was attempted to capture the natural frequencies and corresponding mode shapes. Li at al. [23] have developed a novel dynamic testing procedure to estimate the overall flexural stiffness and accurately evaluate the load carrying capacity of timber bridges. In addition, the procedure is able to identify the defective members of the bridge structure. Peterson et al. [24-26] have applied dynamic system identification to timber structures. The studies showed that the Damage Index method was able to detect and locate the inflicted damage for single damage cases. However, no systematic investigations of the damage index for detecting multiple damage have been reported as yet. Furthermore, evaluation of the severity of damage, quantitatively, in timber structures has not been done to date [27].

A systematic study was therefore conducted to investigate the capabilities and limitations of different damage detection algorithms for locating and evaluating damage inflicted in a timber beam considering single and multiple damage scenarios and reported in this paper. The focus here is on the theoretical and numerical aspects while experimental investigations are presented in the accompanying paper. A numerical model of a timber beam has been developed using finite elements (FE). Modal 
parameters, namely, natural frequencies and mode shapes, were extracted for use in all adopted and extended algorithms. The same model was then inflicted with single and multiple damage for evaluating the capabilities and limitations of different damage detection algorithms in locating the damage. As mentioned earlier, one of the popular and promising damage identification algorithms is DI method, which is based on modal strain energy [28]. However, a systematic investigation of its capability and limitation in locating and quantifying damage in timber structures has been limited. In this paper, after an in-depth exploration of the specific features of damage index and flexibility methods, a modified version of DI is proposed and referred to as Modified Damage Index (MDI) method, which is able to improve the capability of locating damage, especially for multiple damage. A new hybrid algorithm combining MDI and changes in flexibility (CIF) algorithms is also presented for damage severity evaluation, since DI and MDI as well as flexibility are individually unable to provide a meaningful quantification of severity of damage. The contribution of individual mode shapes in damage detection has also been investigated. The numerical results indicate that the proposed Modified Damage Index method has greater advantages compared to the DI and CIF methods, especially for multiple damage cases. The hybrid algorithm based on MDI method and changes in flexibility has enabled evaluation of damage severities.

\section{Finite Element Modelling}

Using a correlated finite element (FE) model, dynamic analysis was performed numerically on a sawn timber beam widely available in Australia. All FE analyses were carried out using a commercial finite element analysis (FEA) package namely, ANSYS.

\subsection{Description of Undamaged Model}


Finite element model of the timber beam without any damage is shown in Figure 1. The specimen's breath and depth were $45 \mathrm{~mm}$ and $90 \mathrm{~mm}$, respectively, with a span length of $4,500 \mathrm{~mm}$. The beam is of radiata pine timber with modulus of elasticity of $12,196 \mathrm{~N} / \mathrm{mm}^{2}$ obtained from a four point bending test. The solid elements (SOLID45 in ANSYS) were utilised to model the beam, for which different damage scenarios can be easily introduced. There are 201 nodes and 200 elements used in the longitudinal direction of the model, denoted by numbers with and without italic, respectively, as shown in Figure 1. In the cross sectional area, there are 20 elements along the height and 4 elements across the width as shown in Figure 2. In order to obtain comparable number of data with the experimental tests in terms of measurement locations, 9 data points with spacings of $1 / 8$ of the span length on the top chord of the beam, including the supports, were used. Five mode shapes were extracted from the FE model as shown in Figure 3. The mode shape data were mass normalised [28], thus the modal vector satisfies the following condition:

$\left\{\phi_{n}\right\}^{T}[m]\left\{\phi_{n}\right\}=1$

where $\left\{\phi_{n}\right\}$ is the normalised modal vector and $[m]$ is the diagonal mass matrix.

\subsection{Simulation of Damage}

The damage to be simulated in this study is pockets of rot or termites attack, typically found in timber bridges. Single and multiple damage scenarios were introduced onto a timber beam that represents a typical girder found in timber bridges. The damage cases as described in Table 1 consisting of a rectangular opening from the soffit of the beam, located at 2/8, midspan (4/8), 5/8 and $6 / 8$ of the span length to simulate pockets of rot or termites attack, which usually starts from the top surface in timber girders, were considered. The damage models were intended to study the 
changes in modal parameters numerically due to different location of damage. In this paper and the discussions that follow, L, M and S denote 'light', 'medium' and 'severe' damage, respectively. All inflicted damage is $1 \%$ of the total span length $(45 \mathrm{~mm})$ in width and consists of cuts of $10 \%, 30 \%$ and $50 \%$ of the beam depth, designating damage cases L, M and S, respectively, as shown in Table 1 . The $10 \%, 30 \%$ and $50 \%$ of the beam depth cut in cross section are corresponding to $27.1 \%, 65.7 \%$ and $87.5 \%$ of the loss of the moment of inertia (I), respectively. The configuration of the damage cases is shown in Figure 4 starting from the soffit of the beam. The method of modelling the damage involves changing the geometry only, and does not introduce nonlinearities into the model. It is, therefore, sufficient to perform a linear analysis to ascertain the impact of damage on the dynamic properties of the structure.

\section{Damage Detection Algorithms}

Firstly, two global damage detection (DD) algorithms were selected to identify the location of inflicted damage in the beam.

\subsection{Damage Index Method}

The damage index method developed by Stubbs et al. [28] and adopted by Peterson [24] was the first algorithm selected to locate the inflicted damage. This method utilises characteristics of mode shape curvature for a beam-like structure as the main variable in the derived damage localisation algorithm based on the relative differences in modal strain energy before and after damage.

Considering a general Euler-Bernoulli beam, the strain energy stored in a system is given by 
$U=\frac{1}{2} \int_{0}^{L} E I\left(\frac{\partial^{2} w}{\partial x^{2}}\right)^{2} d x$

where $E I$ is the flexural rigidity of the beam. An analogy of the energy in modal space associated with $\partial^{2} \phi_{i} / \partial x^{2}$ or $\phi_{i}{ }^{\prime \prime}(x)$ (the second derivative of the $i$ th mode shape $\left(\phi_{i}\right)$ ) is

$U_{i}=\frac{1}{2} \int_{0}^{L} E I\left(\frac{\partial^{2} \phi_{i}}{\partial x^{2}}\right)^{2} d x$

If the beam is subdivided into $N$ elements, then the modal strain energy associated in the $j$ th element for the $i$ th mode is given by

$U_{i}=\frac{1}{2} \int_{j}(E I)_{j}\left(\frac{\partial^{2} \phi_{i}}{\partial x^{2}}\right)^{2} d x$

The fractional energy, denoted by $F_{i j}$, is, therefore, expressed as

$$
\begin{aligned}
& F_{i j}=\frac{U_{i j}}{U_{i}} \text { and } \\
& \sum_{j=1}^{N} F_{i j}=\sum_{j=1}^{N} \frac{U_{i j}}{U_{i}}=1
\end{aligned}
$$

where $F_{i j}<<1$.

Similarly, one can obtain the expression for the fractional energy for the damaged beam, in which a superscript asterisk is used to denote the damaged state. In order to avoid possible singularity problems with the quotient $F i j{ }^{*} / F i j$, shifting of the axis of reference to avoid numerical sensitivities is recommended by Stubbs and Garcia [28]. Thus, the following approximate expression is used, which relates the behaviour of the damaged and undamaged state: 
$1+F_{i j} \approx 1+F_{i j} *$

By choosing the sub-regions to be relatively small, the flexural rigidity for the $j$ th element, $(E I)_{j}$ is roughly constant within the element. The damage indicator, $\beta_{i j}$, for the $i$ th mode may then be defined as

$$
\beta_{i j}=\frac{1+F_{i j}^{*}}{1+F_{i j}}=\frac{\left[\int_{j}\left\{\phi_{i}^{\prime \prime *}(x)\right\}^{2} d x+\int_{0}^{L}\left\{\phi_{i}^{\prime / *}(x)\right\}^{2} d x\right] \int_{0}^{L}\left\{\phi_{i}^{\prime \prime}(x)\right\}^{2} d x}{\left[\int_{j}\left\{\phi_{i}^{\prime \prime}(x)\right\}^{2} d x+\int_{0}^{L}\left\{\phi_{i}^{\prime \prime}(x)\right\}^{2} d x\right]_{0}^{L}\left\{\phi_{i}^{\prime \prime *}(x)\right\}^{2} d x}
$$

It was suggested that each mode shape coordinate in the mode shape matrix be divided by the Euclidean norm of the matrix to obtain a normalised mode shape matrix [24]. The damage index method was then used to compare the normalised mode shape vector for each girder for each of the damage cases versus the corresponding normalised undamaged mode shape vector. To account for all available modes, $N M$, the damage indicator value for a single element $j$ is given as:

$$
\beta_{j}=\frac{\sum_{i=1}^{N M} N_{u m}{ }_{i j}}{\sum_{i=1}^{N M} \text { Denom }_{i j}}
$$

where $\mathrm{NUM}_{i j}=$ numerator of $\beta_{i j}$ and $\mathrm{DENOM}_{i j}=$ denominator of $\beta_{i j}$ in Equation (8), respectively. Transforming the damage indicator values into the standard normal space, normalised damage index $Z_{j}$ is obtained:

$$
Z_{j}=\frac{\beta_{j}-\mu_{\boldsymbol{\beta} \mathbf{j}}}{\sigma_{\boldsymbol{\beta} \mathbf{j}}}
$$


where $\mu_{\beta j}=$ mean of $\beta_{j}$ values for all $j$ elements and $\sigma_{\beta j}=$ standard deviation of $\beta j$ for all $j$ elements. A judgment-based threshold value is selected and used to determine which of the $j$ elements are possibly damaged which in real applications is left to the user to define based on what level of confidence is required for localisation of damage within the structure. For brevity, the details are omitted here and the reader is referred to Stubbs and Garcia [28] or to the cited references for a more thorough derivation of the above equations.

\subsection{Modified Damage Index (MDI) Method}

The damage index method introduced above has been successful in single damage localisation but encountered problems during the identification of multiple damage cases [24-26]. One of the reasons is the fact that Equation (9) accounts for all available mode shapes through the summation of the combination of mode shape curvatures. Although mode shape vectors have been normalised to the Euclidean norm of the matrix, the mode shape curvatures used for the damage index calculation are not normalised. Values of mode shape curvature are dependant on the shapes of each individual mode shape. Instead of reflecting the changes in the curvature due to damage, the summation of non-normalised mode shape curvatures will distort the damage index in favour of higher modes, which results in false damage identifications. To solve this problem, the following algorithm is proposed in this paper: (1) the mode shape vector is normalised with respect to mass; (2) mode shape curvatures for the ith mode of a given beam are normalised with respect to the maximum value of the corresponding mode. After implementing these modifications, Equation (8) is re-written as follows: 


$$
\overline{\beta_{i j}}=\frac{\left[\int_{j}\left\{\bar{\phi}_{i}^{\prime \prime *}(x)\right\}^{2} d x+\int_{0}^{L}\left\{\bar{\phi}_{i}^{\prime / *}(x)\right\}^{2} d x\right] \int_{0}^{L}\left\{\bar{\phi}_{i}^{\prime \prime}(x)\right\}^{2} d x}{\left[\int_{j}\left\{\bar{\phi}_{i}^{\prime \prime}(x)\right\}^{2} d x+\int_{0}^{L}\left\{\bar{\phi}_{i}^{\prime \prime}(x)\right\}^{2} d x\right]_{0}^{L}\left\{\bar{\phi}_{i}^{\prime \prime *}(x)\right\}^{2} d x}
$$

where $\bar{\phi}_{i}^{\prime / *}$ or $\bar{\phi}_{i}^{\prime \prime}$ are normalised curvature vectors. Once again a statistically normalised damage index can be obtained by using equation (10).

\subsection{Flexibility}

The flexibility matrix in the form of modal parameters is presented in Equation (12), adopted from the work of Pandey and Biswas [30]. It should be noted that the mode shapes are also mass normalised.

$$
S=\Phi \Omega^{-1} \Phi=\sum_{i=1}^{n} \frac{1}{\omega_{i}^{2}} \phi_{i} \phi_{i}^{T}
$$

where $\omega_{i}$ is the $i$-th modal frequency, $n$ is the number of degrees of freedom, $\Omega$ is $\omega_{i}^{2}$ and $S$ is the flexibility matrix. The change in flexibility $\Delta$ is defined as the difference of flexibility between the undamaged $\left(S_{u}\right)$ and damaged $\left(S_{d}\right)$ structure as given by

$$
\Delta=S_{u}-S_{d}
$$

For each degree of freedom $j$, let $\left(\delta_{j}\right)_{\max }$ be the maximum absolute value of the elements $\left(\delta_{i j}\right)$ in the corresponding $i$-th column of $\Delta$ as shown in Equation (14).

$$
\left(\delta_{j}\right)_{\max }=\max _{i}\left|\delta_{i j}\right|
$$

The magnitude of $\left(\delta_{j}\right)_{\max }$ is the measure of change in flexibility for each measurement location which is used to detect and locate damage in a structure. 


\subsection{Hybrid of MDI and Flexibility}

A hybrid of Modified Damage Index (MDI) and Changes in Flexibility (CIF) (referred as $\mathrm{HMC}$ in the following discussions) is proposed to estimate severity of damage. The formulation for the HMC is given in Equation (15) and the schematic diagram of the process of obtaining the estimated severity of damage is depicted in Figure 5. This combination is proposed based on the findings that the MDI algorithm was capable of locating the damage but not evaluating its severity, while the magnitude of the CIF algorithm was able to show progressive severity of damage but not performing well in locating the damage. Hence, combining the two algorithms by multiplying the normalised damage indicator of MDI algorithm $\overline{Z_{j}}$ and the CIF algorithm $\left(\delta_{j}\right)_{\max }$, a new indicator is obtained. In order to estimate the severity of damage using the HMC algorithm, a weighting (calibration) process is employed to calibrate the values against single damage scenarios of severe damage condition $(87.5 \%$ loss of 'I' at selected locations of $1 / 8$ to $7 / 8$ of the span length at intervals of $1 / 8$ ). The selected 7 damage locations are deemed sufficient to represent the damage behaviour of a one-dimensional beam system. The HMC values of the selected single damage scenarios were then curve-fitted using the 3-dimensional Gaussian nonlinear regression. The initial outputs of the curve-fitted values were used to estimate the severity of damage in terms of percentage of cut depth from the soffit of the beam. Using the relationship between cut depth and loss of 'I', the initial values were subsequently converted to final output values that can estimate the severity of damage in terms of percentage of loss of 'I'.

$$
H M C=\overline{Z_{j}}\left(\delta_{j}\right)_{\max }
$$




\subsection{Effects of Sensor Density}

The effectiveness of damage localisation algorithms introduced above, is closely related to the number of subregions the given structure or components are divided into, hence determining the number of mode shape coordinates. The number of subregions to be used for damage detection is, in turn, dictated by the number of sensors used in the measurement. In order to produce reliable and accurate damage location, a relatively large number of sensors are required to produce the fine coordinates of the mode shapes. In the numerical simulation, the number of subregions can be easily controlled by mesh density. However, in practical testing, the number of subregions is limited by the number of sensors used in the testing which is often far less than what is desired. To overcome this limitation, a few techniques for reconstructing mode shapes, to increase the number of coordinates, are proposed. In this paper, cubic spline interpolation technique (referred as cubic spline in the following discussions) is used for reconstruction of the mode shapes. The measured mode shape coordinates can be interpolated using this technique to generate mode shape vectors with fine density. A more detailed discussion on the advantage of using cubic spline compared to another mode shape reconstruction algorithm, Shannon Sampling Theorem, can be found in Choi at al. [31].

\section{Analytical Verification of Damage Localisation Algorithm}

In the analytical investigation presented in the following, the statistically normalised damage indicator values $Z_{j}$ for damage index and modified damage index, change in flexibility index and hybrid index for each of the damage cases are plotted against the beam span length. In principle, the index value $Z j$ larger than zero (the 
probability-based criterion for damage) is considered as damage existing at that location. The actual damage locations are indicated with vertical dashed lines in all the figures.

\subsection{Discussions on Damage Index Method}

Figures $6 \mathrm{a}$ and $6 \mathrm{~b}$ show the results of applying damage index (DI) algorithm to detect single damage cases with light damage at location $4 / 8$ or $6 / 8$ of the span length using the first two flexural modes ( 2 modes) for computation, which is commonly used for this damage localisation algorithm. The method gives indications of damage location for both damage cases $4 \mathrm{~L}$ and $6 \mathrm{~L}$. Figures $5 \mathrm{c}$ and $5 \mathrm{~d}$ illustrate the same single damage cases, but using the first five flexural modes (5 modes) in the DI algorithm. Such combination of modes used in damage localisation is not commonly found in the literature. The damage in both cases is being located but a few false detections did also appear, indicated by positive $Z_{j}$ values at locations other than the actual damage.

Figures $7 \mathrm{a}$ and $7 \mathrm{~b}$ show the results of two damage cases (4S6M) using 2 modes and 5 modes in the DI algorithm computation, respectively. From Figure 7a, both severe and medium damage at location $3.375 \mathrm{~m}$ and $2.25 \mathrm{~m}$, respectively, were located but the magnitude of $Z_{j}$ for severe damage is relatively small, which is opposite to the reality. The noted trend is similar to the results found in previous study [24]. In contrast, the 5 mode case as shown in Figure $7 \mathrm{~b}$ shows the severe damage at midspan with higher confidence but misses the medium damage on top of some false positives. Figures 8 and 9 illustrate the results of 3 damage (4S5M6M) and 4 damage (2S4M5M6M) scenarios, respectively. It is apparent, from Figures $8 \mathrm{a}$ and 9a, that the 2 modes used in the DI algorithm computation failed to locate all damage locations as more damage appeared. Similar pattern is also observed in Figures $8 b$ and $9 b$, where 5 modes were used in the 
DI algorithm. Nonetheless, the 5 mode cases have demonstrated some indication of damage locations, which has not been picked up by the 2 mode cases such as damage at location $2.8125 \mathrm{~m}$ for damage case 2S4M5M6M as shown in Figures $8 \mathrm{a}$ and $8 \mathrm{~b}$. This indicates that incorporating higher modes in the DI algorithm may contribute to identifying damage at locations, which is otherwise missed when using just the first two flexural modes.

\subsection{Application of Flexibility on Timber Structures}

In this paper, an attempt has been made to apply CIF in a timber beam, numerically, to detect damage location of single and multiple damage scenarios which have not yet been reported in any publications, specifically with respect to application of CIF with boundary conditions of pin-pin.

For the single damage case $4 \mathrm{~L}$ as shown in Figures 10a and 10c, the change in the flexibility matrix computed using the first two and five flexural modes, respectively, starts with zero at the supports and increases nonlinearly (usually linear in a simply supported case) as it moves towards the midspan of the beam. The algorithm identifies the single damage location at midspan where the changes in flexibility index reaches its maximum as suggested by Pandey and Biswas [30]. The behaviour of CIF in the 4L case is similar to the $6 \mathrm{~L}$ case as shown in Figure 10. The shift of damage location, however, has created another "hump" (local maximum) next to the damage site. This phenomenon was not reported in previous studies for simply supported beam models [30]. The "hump" may be attributed to the pin-ended support which has restricted translational movement resulting in additional forces. It is also important to mention that the number of modes used in the algorithm has not affected the results much. 
The multiple damage scenarios involving cases with two (4S6M), three (4S5M) and four (2S4M5M6M) damage were considered utilising CIF algorithm and using the first two and five flexural modes and they are illustrated in Figures 11 to 13, respectively. The severe damage at midspan of Figures 11 and 12 were identified by the method, but it failed to locate other damage locations. For four damage location cases in Figure 13, the method misses the severe damage as the maximum CIF index corresponds to location $2.8125 \mathrm{~m}$ with medium damage. It is deduced that the CIF method is capable of locating single damage location but does not provide any information on damage regions. Furthermore, it has difficulty identifying all damage locations for multiple damage scenarios.

\subsection{Modification Made to DI Method to Improve Damage Detection}

The DI method can be modified by normalising the mode shape curvature, herewith named Modified Damage Index (MDI) method in this paper. In Figure 14, using the MDI, single damage cases of $4 \mathrm{~L}$ and $6 \mathrm{~L}$ using the first two (2 modes) and five (5 modes) flexural modes to compute are plotted. It is clearly evident that the method is capable of detecting all damage locations. The results also indicated that the method is effective in identifying localised single damage location regardless of the number of modes used, in contrast to the original DI shown in section 4.1. In other words, the MDI method is less dependent on the number of mode shapes selected.

Damage localisation for the case of two damage (4S6M) using either 2 modes or 5 modes in the algorithm are shown in Figures $15 \mathrm{a}$ and 15b, respectively. It can be seen that the MDI method is able to capture all localised damage at locations $2.25 \mathrm{~m}$ and $3.375 \mathrm{~m}$. In these figures, the magnitude of the damage index at the location of damage changes with the number of modes selected for computation. Computing with 5 modes 
shows a reasonable trend in terms of reflecting the probability of damage for either severe or medium damage. In comparison, the 2 mode case merely depicts similar values of damage index for different damage levels. It is therefore confirmed that inclusion of higher modes in the algorithm can produce better or equally good results than that of using only the first two modes. Cases of three and four damage locations (4S5M6M and 2S4M5M6M) are illustrated in Figures 16 and 17. For both cases, when 2 modes are used in the computation, it failed to clearly locate all damage, whereas when 5 modes are used, all damage locations were identified. Therefore, it can be concluded that for multiple damage scenarios employing higher modes in damage localisation with MDI algorithm produces better results. It has been noted that the magnitude of $Z_{j}$ is getting smaller as more damage is introduced into the beam. This may be due to the smaller difference between respective $\beta_{j}$ values at each measuring location along the span length as more damage is inflicted. In fact, the magnitude of $Z_{j}$ indicates the probability of the existence of damage. For instance, in Figure 15b, the two peaks showed a lower index value for medium damage and a higher one for severe damage. Based on this observation, it is possible to estimate that these damages are different in severity, but not in relative values.

\section{Evaluation of the Severity of Damage}

The hybrid of the MDI and CIF methods (HMC) is proposed for estimating the location and severity of damage. In this hybrid algorithm, the level of damage severity represented by percentage of loss of moment of inertia "I" is denoted by horizontal dash-dot lines. Based on the discussion above, the first five flexural modes were used in 
the HMC algorithm. The inflicted and predicted severity of damage is tabulated in Table 2.

The results of single damage scenarios with ascending damage severity are illustrated in Figures 18a to 18f. From these graphs, it is obvious that the method is correctly pin-pointing the damage at locations $2.25 \mathrm{~m}$ and $3.375 \mathrm{~m}$ for damage cases of light (4L and 6L), medium (4M and 6M) and severe (4S and 6S) damage. From Table 2 , the estimation of damage severity for both light damage cases ( $4 \mathrm{~L}$ and $6 \mathrm{~L}$ ) is close to the true value with differences of no more than 5\%. For the medium and severe damage cases at a single location, the difference between the inflicted and predicted severity of damage is even smaller (less than 1\%). It is obvious that the HMC algorithm is very effective in evaluating the severity of single damage scenarios of medium and severe damage, while it predicted reasonably well the light damage with less than $20 \%$ error.

Figures 19 to 21 illustrate the results of HMC algorithm being applied to multiple damage cases. The algorithm has demonstrated again its capability to identify all multiple damage locations for the damage cases discussed here. It also predicted damage severity well for the damage cases of two (4S6M), three (4S5M6M) and four (2S4M6M6M) damage locations with difference of inflicted and estimated severity being less than $15 \%$ as documented in Table 2 . However, the algorithm has relatively high prediction error ranging from $26 \%$ to $87 \%$ for all medium damage in all multiple damage scenarios discussed in this paper. The newly proposed hybrid of modified damage index method and change in flexibility method (HMC) is found to be capable of detecting damage locations and estimating damage severity with high level of accuracy for single damage and at a reasonable level of confidence for multiple damage scenarios. 


\section{Conclusions}

Two damage detection algorithms, namely damage index (DI) method and changes in flexibility (CIF) were adopted to locate and evaluate damage in timber structures. It is found that both algorithms are able to detect single damage location but fail to detect multiple damage scenarios. Therefore, a modified version of the DI algorithm, namely modified damage index (MDI) method is proposed. The MDI algorithm is promising in terms of detecting single and multiple damage scenarios. The modified algorithm is also less dependent on the mode shapes selected compared to its original form for damage detection. A new hybrid algorithm of MDI and CIF algorithms was also proposed to estimate the severity of damage. It is apparent that the hybrid algorithm is a viable tool to assess the severity of damage for single damage scenarios and can predict reasonably well the severity of damage for multiple damage scenarios.

\section{ACKNOWLEDGEMENTS}

The authors wish to thank the Centre for Built Infrastructure Research (CBIR), Faculty of Engineering, at the University of Technology Sydney for supporting this work. The authors would like to express their gratitude to Dr. Ali Saleh for his advice in finite element modelling using ANSYS and to Dr. Joko Widjaja and Mr. Christophe Gerber for sharing their opinions and experiences in many aspects of this study.

\section{References}

[1] Department of Transport and Regional Services, Australia. Chapter 4: Local Government Infrastructure. Local Government National Report, 2003: 61-77. 
[2] RN Emerson, DG Pollock, JA Kainz, KJ Fridley, DI McLean, RJ Ross. Nondestructive evaluation techniques for timber bridges. Proceedings of 1998 World Conference on Timber Engineering (WCTE), Lausanne, Switzerland, 1998: 670-677. [3] RJ Ross, RF Pellerin. Nondestructive testing for assessing wood member in structures: A review. General Technical Report FPL-GTR-70 (Rev.), Madison, WI, US: Department of Agriculture, Forest Service, Forest Products Laboratory, 1993: 40. [4] M Kato, S Shimada. Vibration of PC bridge during failure process. ASCE Journal of Structural Engineering 1986; 112 (7): 1692-1703.

[5] W Ariyaratne, M Al-Dawod, B Samali, A Saleh, S Bakoss. Testing and assessment of a 3-span steel-concrete highway bridge using dynamic methods- A case study. $5^{\text {th }}$ AustRoads Bridge Conference, May 2004: CD-Rom.

[6] DM Armstrong, A Sibbald, MC Forde. Integrity assessment of masonry arch bridges using the dynamic stiffness technique. NDT \& E International 1995; 28 (6): 367-375.

[7] OS Salawu, C Williams. Bridge assessment using forced-vibration testing. ASCE Journal of Structural Engineering 1995; 121 (2): 161-173.

[8] J Maeck, GD Roeck. Damage detection on a prestressed concrete bridge and RC beams using dynamic system identification. Proceedings of DAMAS '99, Dublin, Ireland, June 1999: 320-327.

[9] A Patjawit, W Kanok-Nukulchai. Health monitoring of highway bridges based on a Global Flexibility Index. Engineering Structures 2005; 27 (9): 1385-1391.

[10] T Toksoy, AE Aktan. Bridge-condition assessment by Modal Flexibility. Experimental Mechanics 1997; 34 (3): 271-278. 
[11] M Raghavendrachar, AE Aktan. Flexibility by multireference impact testing for bridge diagnostics. ASCE Journal of Structural Engineering 1992; 118 (8): 2186-2203. [12] J-T Kim, N Stubbs. Improved damage identification method based on modal information. Journal of Sound and Vibration 2002; 252 (2): 223-238.

[13] J-T Kim, N Stubbs. Model-uncertainty impact and damage-detection accuracy in plate girder. ASCE Journal of Structural Engineering 1995; 121 (10): 1409-1417.

[14] S Park, N Stubbs. Bridge diagnostic via vibration monitoring. Proceedings of SPIE- Smart Structures and Materials 1996- Smart Systems for Bridges, Structures and Highways, 28-29 February San Diego, California, US, 1996; LK Matthew (editor): 3645.

[15] J-T Kim. Robust damage localization algorithm for highway plate-girder bridges. Proceedings of SPIE- Smart Structures and Materials 1995- Smart Systems for Bridges, Structures and Highways, 28 February, 2-3 March, San Diego, California, US, 1995; LK Matthew (editor): 117-127.

[16] MN Maduakolam, N Stubbs. Global non-destructive detection of mass and structural damage to conventional and floating bridges. Proceedings of SPIE- Smart Structures and Materials 1995- Smart Systems for Bridges, Structures and Highways, 28 February, 2-3 March, San Diego, California, US, 1995; LK Matthew (editor): 81-95. [17] CR Farrar, DA Jauregui. Comparative study of damage identification algorithms applied to a bridge: II. Experiment. Smart Material Structures 1998; 7: 720-731. [18] JJ Lee, CB Yun. Damage diagnosis of steel girder bridges using ambient vibration data. Engineering Structures 2006; 28: 912-925. 
[19] J Li, B Samali, FC Choi, U Dackermann. Damage identification of timber bridges using vibration based methods. Proceedings of the $11^{\text {th }}$ Asia-Pacific Vibration Conference, Langkawi, Malaysia 2005: 662-668.

[20] CR Farrar, DA Jauregui. Comparative study of damage identification algorithms applied to a bridge: I. Experiment. Smart Material Structures 1998; 7: 704-719.

[21] ML Peterson, RM Gutkowski. Evaluation of the structural integrity of timber bridges. NDT \& E International 1999; 32: 43-48.

[22] A Morison, CD VanKarsen, HA Evensen, JB Ligon, JR Erickson, RJ Ross et al. 2002. Timber bridge evaluation: a global non-destructive approach using impact generated FRFs. Proceedings of IMAC-XX, February 4-7, Los Angeles, California; 2002: 1567-1573.

[23] J Li, B Samali, FC Choi, U Dackermann. Damage identification of timber bridges using vibration based methods. Proceedings of the $11^{\text {th }}$ Asia-Pacific Vibration Conference, Langkawi, Malaysia; 2005: 662-668.

[24] ST Peterson, DI McLean, MD Symans, DG Pollock, WF Cofer, RN Emerson et al. Application of dynamic system identification to timber beam: I. ASCE Journal of Structural Engineering 2001; 127 (4): 418-425.

[25] ST Peterson, DI McLean, MD Symans, DG Pollock, WF Cofer, RN Emerson et al. Application of dynamic system identification to timber beam: I. ASCE Journal of Structural Engineering 2001; 127 (4): 426-432.

[26] ST Peterson, DI McLean, DG Pollock. Application of dynamic system identification to timber bridges. ASCE Journal of Structural Engineering 2003; 129 (1): 116-124. 
[27] C Hu, MT Afzal. A statistical algorithm for comparing mode shapes of vibration testing before and after damage in timbers. Journal of Wood Science 2006; Online First. [28] N Stubbs, J-T Kim, CR Farrar. Field verification of a non-destructive damage localization and severity estimation algorithm. Proceedings of the $13^{\text {th }}$ International Modal Analysis Conference; 1995: 210-218.

[29] RW Clough, J Penzien. Dynamics of Structures $2^{\text {nd }}$ Edition, New York: McGraw-Hill, 1993.

[30] AK Pandey, M Biswas. Damage detection in structures using changes in flexibility. Journal of Sound and Vibration 1994; 169 (1): 3-17.

[31] FC Choi, J Li, Samali B, Crews K. Impact of different numerical techniques on damage identification in structures. The Tenth East Asia-Pacific Conference on Structural Engineering and Construction, 3-5 August, Bangkok, Thailand, 2006: 111116. 


\title{
Application of the Modified Damage Index method to timber beams - II (experimental studies)
}

\author{
F.C. Choi, J. Li, B. Samali* and K. Crews \\ Center for Built Infrastructure Research, Faculty of Engineering, University of Technology Sydney \\ PO Box 123 Broadway, NSW 2007, Australia
}

\begin{abstract}
As the second part of the two companion papers, in this part, the damage detection algorithms theoretically investigated in the companion paper, are subjected to experimental investigation. Experimental Modal Analysis (EMA) were conducted after modal testing to obtain modal results that monitor changes in modal strain energy between the mode shapes of a damaged beam and its corresponding undamaged state. An experimental laboratory experiments on pin-pin supported timber beams are presented in this paper. The capabilities and limitations of the proposed methods are discussed. The first five flexural modes from the Experimental Modal Analysis were used in the computation of damage based on the findings from the analytical investigation presented in the companion paper. Experimental investigations confirm that the modified damage index algorithm provides better results in detecting single and multiple damage scenarios compared to its original form. It is also less dependent on the number of modes selected in the computation and can detect damage with a higher degree of confidence. With the use of experimental results, the proposed hybrid algorithm, which combines the modified index method and changes in flexibility algorithms, is able to evaluate the severity of damage reasonably well when serious

\footnotetext{
* Corresponding author. Tel.: +61 2 95142023; fax: +61 295142868.

E-mail addresses: Bijan.Samali@uts.edu.au
} 
damage was inflicted. This is due to timber’s inherent natural variability within itself. The proposed algorithms are viable tools to identify the location of damage as well as to estimate the severity of localised damage in the form of pockets of rot in timber structures.

Keywords: Damage localisation; severity estimation; timber beam; experimental modal analysis; modified damage index.

\section{Introduction}

Damage detection and structural health monitoring of bridge structures, which increasingly experience deterioration, aging and traffic overloads, have attracted a great deal of attention among researchers. Among various proposed damage detection algorithms, the methods based on vibration of structures have shown great potential. However, very little work has been reported on timber structures such as timber bridges using such methods [1-4]. The fact that even in Australia alone, there are approximately 27,000 aging timber bridges [5], justifies development of a systematic and global damage inspection, identification and health monitoring schemes for integrity/health monitoring of these built infrastructures.

It has been a challenge to apply global nondestructive evaluation (NDE) techniques in wood products such as a timber beam, with inherent natural variability in material properties within the sample itself, especially when using a global nondestructive damage detection tool such as vibration-based methods. In the past decade, many methods of damage detection, based on vibration techniques, have been 
developed for various engineering structures. Some of these methods have shown to be applicable to steel and concrete bridges [6-8].

The accompanying paper [9] has described numerical investigation of a pin-pin supported timber beam using vibration method for the damage detection (DD). The systematic investigation adopted two damage detection algorithms, namely, damage index (DI) and changes in flexibility (CIF) methods, using single and multiple damage scenarios, which provided valuable insight into capabilities and limitations of these damage localisation algorithms. These motivated the development of a modified damage index (MDI) algorithm for locating damage and a hybrid algorithm by combining MDI and CIF algorithms as well as incorporating a curve-fitting process to evaluate severity of damage.

This paper presents experimental investigations on timber beams using a vibration based method, namely experimental modal analysis (EMA) to detect damage. Similar damage detection algorithms to those used in the numerical investigation were used to locate and evaluate various damage scenarios in the experimental work except the changes in flexibility (CIF) method. A thorough laboratory investigation was conducted on timber beams inflicted with various damage scenarios. The modal parameters obtained from the undamaged and damaged state of the test beams were used in the computation of modal strain energy and flexibility. A statistical approach was also adopted to detect location of damage. A mode shape reconstruction technique (cubic spline interpolation) was used to enhance the capability of the damage detection algorithm with limited number of sensors. 


\section{Description of test samples}

\subsection{Undamaged Beams}

Two timber beams were used in the experimental work, representing the scaled bridge girders by maintaining dynamic similitude between test girders and typical timber bridges in Australia. These two beams were designated as Beam1 and Beam2. The basic dimensions of the beams are shown in Figure 1. The beams are of treated radiata pine sawn timber measuring nominal dimensions of $45 \mathrm{~mm}$ by $90 \mathrm{~mm}$ in cross section with a span length of 4,500mm. A specially designed support system was used to support the beam and provide a well-defined boundary condition that is very close to an idealised pin-pin condition as depicted in Figure 2.

\subsection{Inflicted Damage in Beams}

The goal of this study was to detect damage typically found in timber bridges. It is intended to locate damage which occurred in single and multiple locations on a timber beam. The damage cases are described in Table 1. The damage cases described here are only subsets of those in the companion paper [9] due to the fact that it is too time consuming to repeat all numerical damage scenarios in the experimental work. All induced damage scenarios consist of a rectangular opening along the span and from the soffit of the beam, located at 2/8, midspan (4/8), 5/8 and 6/8 of the span length to simulate pockets of rot, which usually starts from the top surface in timber girders. In this paper and the discussions that follow, L, M and S denote 'light', 'medium' and 'severe' damage, respectively. All inflicted damages are $1 \%$ of the total span length and consist of $10 \%, 30 \%$ and $50 \%$ of the beam depth, designating damage cases $\mathrm{L}, \mathrm{M}$ and S, respectively as shown in Table 1 . The $10 \%, 30 \%$ and $50 \%$ loss of the beam depth in 
cross section correspond to $27.1 \%, 65.7 \%$ and $87.5 \%$ of loss of 'I' (moment of inertia), respectively. The configuration of the damage cases is shown in Figure 3.

\section{Experimental Modal Analysis}

The Experimental Modal Analysis (EMA) procedure and instrumentation layout used during the modal tests are shown in Figure 4. The modal testing and EMA in this study has been used to obtain experimental modal parameters such as natural frequencies and corresponding mode shapes. The modal testing employs an impact hammer to excite the test sample at a strategic location and measures the acceleration response. Nine accelerometers were used to measure the acceleration response of the beam, which is deemed as sufficient number of points along the span so that the mode shapes can be accurately reconstructed using interpolation techniques. One of the accelerometers was used for driving point measurement, so that the experimental mode shape can be mass normalised. In order to install the accelerometers to the test sample, each accelerometer was attached onto a small steel plate using magnetic base and secured onto the top chord of the girders. The nine accelerometers were located at every 1/8 intervals of the span length starting from one end support of the beam to the other

end as shown in Figure 1. The impact location was selected at 3/4 of the span length so that more vibrational modes can be excited, simultaneously.

A HP VXI data acquisition system equipped with LMS software was used to record the dynamic response at 10,000 Hz sampling rate yielding 8,192 data points. LMS modal analysis software is used for extracting modal parameters from the measured data in which frequency domain direct measurement curve-fitting technique was used. From the experimental tests and EMA, five vibrational modes with a 
frequency bandwidth from $10 \mathrm{~Hz}$ to $200 \mathrm{~Hz}$, were captured. The Frequency Response Function (FRF) of the driving point is shown in Figure 5. It should be noted that the results for the fourth flexural mode (mode 4) was not satisfactory because the impact location falls on one of its nodes. Nevertheless, this mode was still used in the subsequent discussions. In order to perform accurate damage localisation, the nine-point experimental mode shapes were reconstructed to 41 point mode shapes using cubic spline interpolation technique. The mode shape reconstruction technique enables refinement of mode shape coordinates from the limited number of measurements, therefore enabling prediction of finer damage locations using various damage detection algorithms.

\section{Results and Discussion}

In the following, all damage indices (damage indicators) for each damage case are plotted against span length of the beam. In principle, a positive index value $Z j$ (the probability-based criterion for damage) represents damage at a given location. For the estimation of damage severity, in terms of percentage of loss in 'I', the hybrid algorithm (HMC) of modified damage index (MDI) and changes in flexibility (CIF) algorithms is used. For ease of comparison, the actual damage locations are indicated with vertical dashed line, while horizontal dash-dotted lines indicate inflicted severity of damage in all figures.

\subsection{Discussions on the Effects of Sensor Density}

From Figure 6a, it is clear that without sufficient number of measurements, the damage detection algorithm may not produce reliable damage locations as seen by the circled false detection. Considering the practicality of limited number of sensors in field 
testing, in order to produce sufficiently fine modal coordinates, mode shapes reconstruction is an important part of the damage localisation process. From Figure 6b, it is obvious that the cubic spline interpolation technique has improved detection results with respect to the two true damage locations by eliminating the false damage locations which occur when the original mode shape was used for damage detection (Figure 6a). In the following discussion, the cubic spline interpolation technique will be used for reconstruction of mode shapes from the given data (9 data points for each beam in this study).

\subsection{Damage Index Method}

Illustrated in Figure 7 are the single damage cases in which the damage index (DI) method has been applied for damage localisation using the first two and the first five flexural modes, namely 2-modes and 5-modes, respectively. For single damage cases using 2-modes for DI computation, the damage indicator is able to indicate the damage locations with few false positives (indication of false damage locations) as shown in Figures 7c, 7e and 7g (with the exception of the case shown in Figure 7a). As depicted in Figures 7b and 7d, using DI method with 5-modes for the light damage cases, the damage indicator fails to locate the damage. Nevertheless, for the medium and severe cases, using DI method with 5-modes (Figures $7 \mathrm{f}$ and $7 \mathrm{~h}$ ), the damage locations are detected quite clearly despite many appearances of false positives. It is obvious that using higher modes when using this algorithm provides better results than just the first two modes.

The results of applying DI algorithm for multiple damage scenarios are shown in Figures 8 to 10. The results of using 2-modes and 5-modes when applying the algorithm were consistent with the findings of the analytical study for all multiple damage cases 
[9], namely, using 2-modes failed to locate all damage locations and using 5-modes detected all damage locations as shown in Figure 10. The original DI algorithm is very poor for damage detection when applied to experimental data, especially for multiple damage cases.

\subsection{Modified Damage Index Method}

By normalising the mode shape curvature, one can improve the functions of the original damage indicator algorithm (damage index). From Figure 11, it can be seen that for single damage scenarios using modified damage index (MDI) algorithm using either 2-modes or 5-modes in the calculation, the minimum damage that could be comfortably detected is medium damage ( $65.7 \%$ loss of 'I') or more severe damage. The light damage is also located but is overwhelmed by false positives (indication of damage that does not exist). The reason is that the modal parameters for light damage (27\% loss in 'I’) are more susceptible to noise influence, hence hampering the possibility of being detected with the modified localisation algorithm in single damage cases.

For the multiple damage scenarios as depicted in Figures 12 to 14, besides usual noise in the experimental modal analysis data, the mode shape and its derivatives are also altered in larger scale as more damage is inflicted and the spacing between damage is getting closer. This may have suppressed the contribution of light damage in the damage detection, which is quite obvious for medium damage in Figure 13. Employing the MDI method with 5-modes in the computation still allows the detection of all damage locations in the cases discussed here compared to using only 2-modes as illustrated in Figure 14. It is apparent that the MDI performs better in terms of damage localisation using higher modes in the calculation compared to selecting only the first two modes. The modified algorithm is also less dependent on the mode shapes selected. 


\subsection{Evaluation of Damage Severity Using Hybrid of MDI and CIF Methods}

As mentioned in the accompanying paper, the MDI and DI methods are capable of detecting location of damage, but unable to evaluate the severity of damage. Hence, combining the MDI and CIF and incorporating curve-fitting of various single damage scenarios provide a way (abbreviated as HMC method) that could estimate the severity of damage. However, using the experimental modal analysis data that contains noise, the method requires small adjustment to overcome measurement error resulting from noise pollution.

After minor adjustment, the results for light damage (27.1\% loss in ' $\mathrm{I}$ ') for single damage scenarios at location 2.25m and 3.375m are shown in Figures 15a and 15b, respectively, and the extent of damage is tabulated in Table 2. These figures show that the HMC algorithm is able to identify the location of damage but with other overwhelming false positives. From Table 2, the predicted damage severity for cases $4 \mathrm{~L}$ and $6 \mathrm{~L}$ produces an error of more than $100 \%$. For the medium and severe damage at midspan (4M and 4S) as illustrated in Figures 15c and 15d, the location and severity of damage are being detected quite accurately with less than $7 \%$ error in predicting the severity of damage. This implies that the HMC method is capable of estimating severity of damage as well as location of damage for medium or higher damage but it requires more care when applied in light damage scenarios.

Figures 16 to 18 show the evaluation of damage severity using the HMC method for damage cases of two (4S6M), three (2M4M6S) and four (2S4S5S6S) damage 
locations, respectively, and the magnitude of severity of damage is documented in Table 2. These figures demonstrate that all damage locations for the multiple damage scenarios are identified using the MDI algorithm computed with 5-modes. From Table 2, the algorithm is also capable of evaluating severity of damage for severe damage (87.5\% loss in 'I') with errors less than 7\%. For the medium damage (65.7\% loss in 'I'), it exhibits higher errors of about $25 \%$ in some cases. Nevertheless, the method is able to identify damage locations quite accurately and the shortcomings of the method can be compensated with other NDE techniques, if necessary.

\section{Conclusions}

Following the numerical investigation on the FE beam models in the companion paper, this experimental investigation was set to systematically explore capabilities and limitations of various damage localisation algorithms proposed in the companion paper in terms of detecting and locating damage in timber beams. First, the algorithms were verified numerically using a finite element (FE) model in the companion paper.

The laboratory tests of timber beams were carried out with pin-pin supports representing the actual timber bridge supports. Meanwhile, timber beams were replica of girders in timber bridges, which are structurally more complex. Damage was inflicted onto the test samples to simulate pockets of rot in timber structures at single or multiple locations with various severities. The modal parameters were obtained from the modal testing and experimental modal analysis. It was found that using the original experimental data, the Damage Index (DI) algorithm produced poor results with respect to damage identification, especially for multiple damage cases. However, the proposed Modified Damage Index (MDI) algorithm produced much better results for both single 
damage and multiple damage cases, especially for medium and severe damage cases. The proposed hybrid algorithm performed well in locating and evaluating the severity of damage.

Overall, it can be concluded that for localisation and evaluation of damage in timber beam/bridge, the proposed modified DI and hybrid algorithm provide reasonably reliable and accurate tools for damage detection of medium to severe damage.

\section{ACKNOWLEDGEMENTS}

The authors wish to thank the Centre for Built Infrastructure Research (CBIR), Faculty of Engineering, University of Technology Sydney, for supporting this work. Within the Faculty of Engineering, The authors wish to also thank the staff of UTS Structures Laboratory for their assistance in conducting the experimental works.

\section{References}

[1] ST Peterson, DI McLean, MD Symans, DG Pollock, WF Cofer, RN Emerson et al. Application of dynamic system identification to timber beam: I. ASCE Journal of Structural Engineering 2001; 127 (4): 418-425.

[2] ST Peterson, DI McLean, MD Symans, DG Pollock, WF Cofer, RN Emerson et al. Application of dynamic system identification to timber beam: I. ASCE Journal of Structural Engineering 2001; 127 (4): 426-432.

[3] ST Peterson, DI McLean, DG Pollock. Application of dynamic system identification to timber bridges. ASCE Journal of Structural Engineering 2003; 129 (1): 116-124. 
[4] A Morison, CD VanKarsen, HA Evensen, JB Ligon, JR Erickson, RJ Ross et al. 2002. Timber bridge evaluation: a global non-destructive approach using impact generated FRFs. Proceedings of IMAC-XX, February 4-7, Los Angeles, California; 2002: 1567-1573.

[5] K Crews, B Samali, S Bakoss, C Champion. Overview of assessing the load carrying capacity of timber bridges using dynamic methods. $5^{\text {th }}$ Austroads Bridge Conference May 2004, Tasmania: CD-Rom.

[6] JJ Lee, CB Yun. Damage diagnosis of steel girder bridges using ambient vibration data. Engineering Structures 2006; 28: 912-925.

[7] A Patjawit, W Kanok-Nukulchai. Health monitoring of highway bridges based on a Global Flexibility Index. Engineering Structures 2005; 27 (9): 1385-1391.

[8] MK Yoon MK, D Heider, Jr JW Gillespie, CP Ratcliffe, RM Crane. Local damage detection using a global fitting method on mode shape data. Proceedings of the IMAC-XIX, Kissimmee, Florida, US, 2001: 231-237.

[9] FC Choi, J Li, B Samali B, K Crews. XXXX. XXX, 200X: the companion paper. 